\title{
Warehouse Design and Planning: A Mathematical Programming Approach
}

\author{
Carla A.S. Geraldes ${ }^{1}$, Maria Sameiro Carvalho ${ }^{2}$, and Guilherme A.B. Pereira ${ }^{2}$ \\ ${ }^{1}$ Centro ALGORITMI and Polytechnic Institute of Bragança, Portugal \\ carlag@ipb.pt \\ 2 Centro ALGORITMI and University of Minho, Portugal \\ sameiro@dps.uminho.pt, guilherme.pereira@algoritmi.uminho.pt
}

\begin{abstract}
The dynamic nature of today's competitive markets compels organizations to an incessant reassessment in an effort to respond to continuous challenges. Therefore, warehouses as an important link in most supply chains, must be continually re-evaluated to ensure that they are consistent with both market's demands and management's strategies. A number of warehouse decision support models have been proposed in the literature but considerable difficulties in applying these models still remain, due to the large amount of information to be processed and to the large number of possible alternatives. In this paper we discuss a mathematical programming model aiming to support some warehouse management and inventory decisions. In particular a large mixed-integer nonlinear programming model (MINLP) is presented to capture the tradeoffs among the different inventory and warehouse costs in order to achieve global optimal design satisfying throughput requirements.
\end{abstract}

Keywords: Supply chain management, Warehouse models, Inventory management, Mathematical modelling.

\section{Introduction}

Market competition requires continuous improvement in the design and operation of supply chains. A supply chain can be considered as a network of entities whose efficiency and effectiveness is highly determined by the performance of the overall network (see Fig. 11).

In a supply chain network, products need to be physically moved from one location to another. During this process, they may be buffered or stored at certain facilities (warehouses) for a certain period of time for strategic or tactical reasons. Within this context, warehouses play an important role in supply chains and are a key aspect in a very demanding, competitive and uncertain market.

On the other hand, modern supply chain principles compel companies to reduce or eliminate inventory levels. Additionally warehouses require capital, labour, and information technologies, which are expensive resources. So, why do we still need warehouses? 


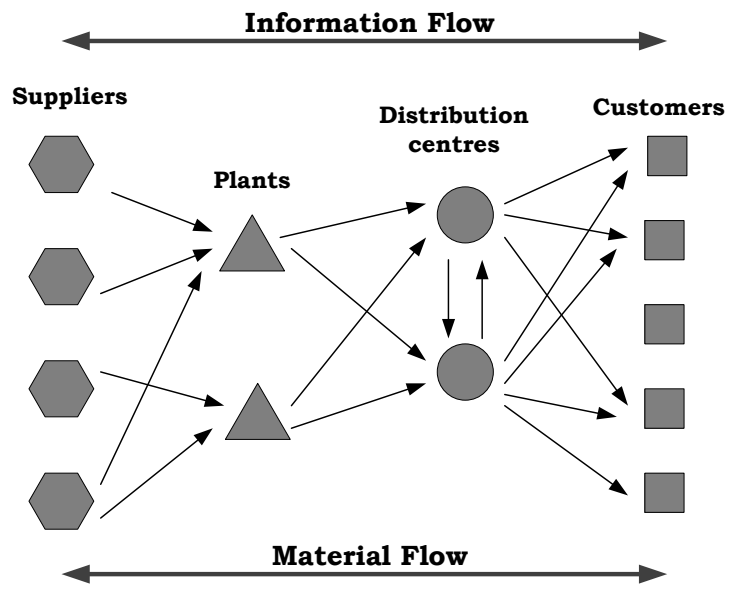

Fig. 1. A typical supply chain network

According to Bartholdi and Hackman [1] there are four main reasons why warehouses are useful:

1. To consolidate products in order to reduce transportation costs and provide customer service;

2. To take advantage of economies of scale;

3. To provide value-added processing services, and

4. To reduce response time.

Thus, warehouses will continue to be an important node at the logistic network by the fact that if a warehouse cannot process the orders quickly, effectively, and accurately, then all the supply chain optimization efforts will suffer (see Tompkins [12]).

In distribution logistic where market competition requires higher performances from the warehouses, companies are compelled to continuously improve the design and planning of warehouse operations. Furthermore, the ever increasing variety of products, the constant changes in customer demands and the adoption of agile management philosophies also bring new challenges to reach flexible structures that provide quality, efficiency and effectiveness to the logistics operations.

Some major decisions involved in the warehouse design and operation problems are illustrated in Fig. 2 (see Gu et al. 44). Warehouse design and planning decisions typically run from a functional description, through a technical specification, to equipment selection and determination of the layout. The overall structure decision determines the material flow patterns within the warehouse, the specification of the functional areas and the flows between the areas. Sizing and dimensioning decisions determine the total size of the warehouse as well as the space allocation among functional areas. Layout definition is the detailed configuration within a functional area and equipment decisions define 


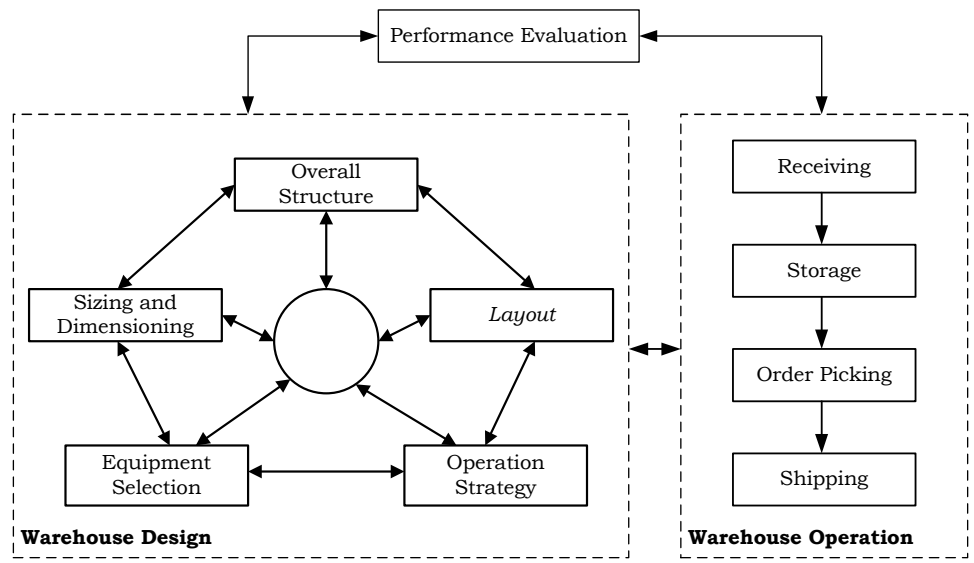

Fig. 2. A framework for design and operation problems (adapted from Gu et al. 4]

an automation level for the warehouse and identify equipment types. Finally operating policies refer to storage, picking and routing decisions.

Hassan [7] presented a framework for the design of a warehouse. The proposed framework accounts for several factors and operations of warehousing such as:

1. Specification of warehouse type and purpose;

2. Analysis and forecasting of the demand;

3. Definition of operating policies;

4. Establishment of inventory levels;

5. Class formation;

6. Definition of functional areas and general layout;

7. Storage partition;

8. Selection of equipment for handling and storage;

9. Design of aisles;

10. Determination of space requirements;

11. Location and number of Input/Output points;

12. Location and number of docks;

13. Arrangement of storage;

14. Zone formation.

Once these warehouse decisions are strongly interrelated, warehouse design is a highly complex task where conflicting objectives impose specific trade-offs.

Despite the various decision models available in scientific literature, the majority addresses isolated or simplified problems in order to provide the best solution. However, most of the real problems are unfortunately not well-defined and often cannot be reduced to multiple isolated sub-problems. Therefore, warehouse design often requires a mixture of analytical skills and creativity. Anyhow, research aiming an integration of various decisions models and methods is badly 
needed in order to develop a methodology for systematic warehouse design (see Rouwenhorst et al. [10]).

Furthermore, as the literature review in next section shows, most research efforts have been dedicated to warehouse operations decisions instead of design decisions. This is not surprising since design decisions models are more difficult to develop and treat analytically once they require the integration of several and complex issues.

In this paper we present a warehouse and inventory mathematical model that jointly integrates issues concerning:

- The size of the warehouse;

- The external storage additional capacity, if needed;

- The replenishment quantities and reorder points of products to be stored.

Our aim is to test an integrated approach that takes into account inventory and some warehouse design decisions.

In the Section 2 of this paper we will present a brief literature review on warehouse design and planning issues. The purpose of this section is not limited to the specific studied problem but also covers other important topics in warehouse literature. The Section 3 will present the proposed warehouse sizing and inventory model formulation and a description of the methodology used to solve the model. Computational results will be presented and summarized in Section 4 , and finally some conclusions and future work directions are reported in Section 5

\section{Literature Review}

Warehousing is concerned with all the material handling activities that take place within a warehouse. It includes the receiving of products, storage, order-picking, accumulation, sorting and shipping operations. Basically, one can distinguish two types of warehouses: distribution warehouses and production warehouses. According to Van den Berg and Zjim [14, a distribution warehouse is a warehouse in which products from different suppliers are collected (and sometimes assembled) for delivery to a number of customers. On the other hand, a production warehouse is used for the storage of raw materials, semi-finished products and finished products in a production facility.

There are many activities that occur at a warehouse. Typically, distribution warehouses receive products - Stock Keeping Units (SKUs) - from suppliers, unload products from the transport carrier; store products, receive orders from customers, assemble orders, repackage SKUs and ship them to their final destination. Frequently, products arrive packaged on large scale units and are packaged and shipped on small units. For example, SKUs may arrive in full pallets but must be shipped in cases. Fig. 3 shows the typical functional areas and flows within warehouses.

At the receiving area products (or items) are unloaded and inspected to verify any quantity and quality inconsistency. Afterwards, items are transferred 


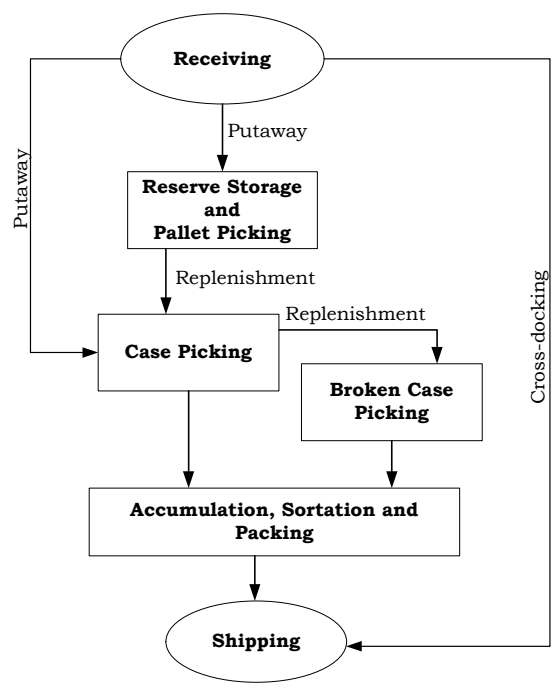

Fig. 3. Typical warehouse functions and flows

to a storage zone or are directly placed to the shipping area (this is called a cross-docking operation). We can distinguish two types of storage areas: reserve storage area and forward or picking area. The reserve area is the place where the products stay until they are required by costumers' orders. The picking area is a relatively small area, typically used to store fast moving products. Most of the flows between these areas are the result of replenishment processes. Order picking is one of the most important functions in most warehouses. SKUs are retrieved from their storage positions based on customers' orders and moved to the accumulation and sorting area or directly to the shipment area. The picked units are then grouped by customers order, packaged and stacked on the right unit load and transferred to the shipping area.

\subsection{Warehouse Design and Planning}

Warehouse design can be defined as a structured approach of decision making at distinct decision levels in an attempt to meet a number of well-defined performance criteria. At each level, multiple decisions are interrelated and therefore it is necessary to cluster relevant problems that are to be solved simultaneously. According to Rouwenhorst et al. [10] a warehouse design problem is a "coherent cluster of decisions" and they define decisions to be coherent when a sequential optimization does not guarantee a globally optimal solution.

The design of a warehouse is a highly complex problem. It includes a large number of interrelated decisions involving warehouse processes, warehouse resources and the organization of the warehouse (see Heragu [8]). Rouwenhorst et al. 10] 
classify the management decisions concerning warehousing into strategic decisions, tactical decisions and operational decisions. Strategic decisions are long term decisions and always mean high investments. The two main issues are concerned with the design of the process flow and with the selection of the types of the warehousing systems. Tactical management decisions are medium term decisions based on the outcomes of the strategic decisions. The tactical decisions have a lower impact than the strategic decisions, but still require some investments and should therefore not be reconsidered too often. At the operational level, processes have to be carried out within the constraints set by the strategic and tactical decisions made at the higher levels. In this level, the concern includes the operational policies such as storage policies and picking and routing operations.

After determining warehouse location and its size, layout decisions must include areas definition and what size should be allocated to each functional area. The forward-reserve problem (FRP) is the problem of assigning products to the functional areas. In this problem the critical decision concerns the choice of products that will be stored in the forward area. Van den Berg et al. [13] proposed a binary programming model to solve the FRP in the case of unit load replenishment, and presented efficient heuristics that provide tight performances guaranties. These replenishments can occur during busy or idle picking periods. The objective was to minimize the number of urgent or concurrent replenishments of the forward area during the busy periods. Although addressing this problem is a strategic decision problem, it is strongly associated upon some tactical problems such as how the items will be distributed among the functional areas. However, the approach usually adopted is to solve the problems sequentially by generating multiples alternatives for the functional area size problem and then determine how the products can be allocated for each of the alternatives.

Gray et al. 5] developed an integrated approach for the design and operation of a typical order-consolidation warehouse. This approach included warehouse layout, equipment and technology selection, item location, zoning, picker routing, pick generation list and order batching. Due to the complexity of the overall problem, they developed a multi-stage hierarchical decision approach. This hierarchical approach used a sequence of coordinated mathematical models to evaluate the major economic trade-offs and to reduce the decision space to a few number of alternatives. They also used simulation technique for validation and fine tuning of the resulting design and operating policies.

Heragu et al. 8 developed a mathematical model and a heuristic algorithm that jointly determines the size of the functional areas and the allocation of the product in a way that minimizes the total material handling and storage costs. The proposed model uses real data readily available to a warehouse manager and considers realistic constraints.

Geraldes et al. 2] adapted the mixed-integer programming model proposed by Heragu et al. 8 to tackle the storage allocation and assignment problems during the redesign process of a Portuguese company warehouse. 
More recently Strack and Pochet [1] presented a robust approach that integrates aspects such us: (i) the size of the functional areas; (ii) the assignment and allocation of products to storage locations in the warehouse; (iii) the replenishment decision in the inventory management. This is probably the most integrated decision model found in this area, nevertheless still assumes fixed and known capacity for the warehouse.

\subsection{Inventory Decisions}

The adoption of new management philosophies compels companies to eliminate or reduce inventory levels. In addition to warehouse management decisions, an appropriate inventory policy may result in a reduction of the total warehousing costs and can also improve the efficiency of the operating policies within the warehouse. The aim of inventory management is to minimize total operating costs satisfying customer service requirements (see Ghiani et al. 3]). To accomplish this, an optimal ordering policy must answer questions, for each SKU, such us when to order and how much to order.

Two different inventory policies arise (see Hadley and Whitin [6]): continuous review policy and the periodic review policy. The first policy implies that the stock level will be monitored continuously. Whenever the inventory on hand decreases to a predetermined level, referred to as the reorder point, a new order is placed to replenish the inventory level. The placed order is a fixed quantity that minimizes the total inventory costs and is normally called the economic order quantity. In the second policy, the inventory level is checked at specific fixed time periods and an order decision is made to complete the stock to a desired upper limit. In this system the inventory level is not monitored at all during the time interval between orders, so it has the advantage of little or no required record keeping. The disadvantage is less direct control. Such system also requires that a new order quantity must be determined each time a periodic order is made. The operating costs taken into account in both inventory policies are the acquisition cost, the holding cost and the shortage cost.

These basic policies can be adapted to take into account special situations such us single or multi-item models with or without a constraint on the total storage space, deterministic or stochastic demands, lost sales, etc. For more details and examples see Ghiani et al. 3 and Nahmias 9 .

\section{Mathematical Programming Model}

Storage may be considered one of the major warehouse functions. Some fundamental decisions occur during the design and planning of a warehouse: (i) how much inventory should be kept in the warehouse for each SKU; (ii) how frequently and at what time should the inventory for a SKU be replenished, and (iii) what should be the size of the warehouse.

The first two decisions lead to the traditional inventory management problem and the last one is probably one of the most important aspect in designing a 
warehouse. Once warehouse size is determined, it will act as a constraint that may last for a long period of time.

Generally there are two basic choices for warehousing. The first is to operate a owned (or private) warehouse and the second is to rent storage space from a public warehouse. Depending on which is least expensive a warehouse manager may use only one type or adopt a mixed strategy - both owned and rented storage space.

In this section we present a mathematical programming model that integrates the warehouse sizing problem and the inventory decisions. The mathematical model determines the optimal overall size for a warehouse in a way that minimizes the inventory and warehouse costs. In developing the model we also assume that external storage space is available to rent to allow the selection of the most adequate storage alternative: storage space ownership, rented warehouse space or a combined solution. Furthermore, an inventory control policy will be based on continuous review policy (reorder point system).

The problem can be stated as follow: a distribution company delivers a set of $i$ products to its customers. Customers demand is well defined and unit acquisition, processing, storage and inventory carrying costs are known. Given warehousing costs (both own storage space and rented space) the objective is to determine the size and type (own or rented) of storage space required as well as the quantity of each product to order and respective reorder point, for a level of service and that minimizes total system costs.

\subsection{A MINLP Model for the Warehouse Design Problem}

The following notation, adapted from Strack and Pochet [1], is used:

Parameters:

$i \quad$ : Product number $(i=1, \ldots, I)$

CostCar : Inventory carrying cost

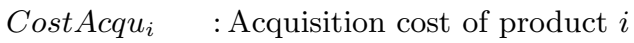

CostShort : Shortage cost

CostRecp : Reception cost

CostCapaS : External capacity cost

CostCapaW : Capacity cost of the private warehouse

CostCapaFW : Private warehouse capacity fixed cost

$E\left(U_{i}\right) \quad$ : Expected value of the demand of product $i$

$L \quad$ : Supply lead time

$d_{i}^{L} \quad$ : Demand of product $i$ during $L$

$\mu_{i}^{L} \quad$ : Average demand of product $i$ during $L$

$\sigma_{i}^{L} \quad$ : Standard deviation of demand of product $i$ during $L$

$M \quad$ : Large positive number 
Decision variables:

$$
w= \begin{cases}1 \text { if we have a private warehouse } \\ 0 & \text { otherwise }\end{cases}
$$

CapaW : Capacity of the private warehouse

CapaS : External additional storage capacity

$Q_{i}$ : Replenishment quantity of product $i$

$r_{i}$ : Reorder point of product $i$

The general formulation of the model can be stated as:

$$
\begin{aligned}
\text { minimize } & \sum_{i=1}^{I} \text { Costcar } \times\left(\frac{Q_{i}}{2}+r_{i}-\mu_{i}^{L}\right)+\sum_{i=1}^{I} \text { CAcqu } u_{i} E\left(U_{i}\right) \\
+ & \sum_{i=1}^{I} \text { CostShort } \times \frac{E\left(U_{i}\right)}{Q_{i}} \times \int_{r_{i}}^{\infty}\left(d_{i}^{L}-r_{i}\right) f\left(d_{i}^{L}\right) \mathrm{d} d_{i}^{L} \\
+ & \sum_{i=1}^{I} \operatorname{CostRecp} \times \frac{E\left(U_{i}\right)}{Q_{i}}+\text { CostCapaS } \times \text { CapaS } \\
+ & \text { CapaW } \times w \times(\text { CostCapaW }+ \text { CostCapaFW }),
\end{aligned}
$$

subject to:

$$
\begin{aligned}
& \sum_{i=1}^{I}\left(Q_{i}+r_{i}-\mu_{i}^{L}\right) \leq \text { CapaW }+ \text { Capa } S, \\
& C a p a W \leq M w, \\
& C a p a W \geq w, \\
& Q_{i}, r_{i} \geq 0, \\
& \text { CapaW, CapaS } \geq 0, \\
& w \in\{0,1\} .
\end{aligned}
$$

The objective function (11) is the expected warehouse and inventory costs per period. Concerning the inventory costs we have taken into account: carrying cost, acquisition cost and shortage cost. The warehouse costs are composed by the reception cost, the additional external storage capacity cost and the costs of the private warehouse. The integrity of the model is ensured by the capacity constraint (2) that guaranties that the required storage capacity is met. Constraints (3)-(4) serve to include the costs of the private warehouse. Finally, a set of variables must be nonnegative (5)-(6) and another is considered binary (7). The above model considers inventory and warehouse size decisions since it integrates both issues supporting decision makers defining the best warehousing alternative, taking into account space requirements determined by customers' demand. 


\subsection{Methodology}

The above model jointly integrates inventory decisions and size decisions (both ownership and rented storage space). It is a mixed-integer nonlinear programming model with a large number of variables when real cases are considered.

To evaluate the computational performance involved in solving the proposed integrated model, experimental tests were performed using LINGO 12.0 solver which uses branch-and-bound algorithm for integer nonlinear problems. All tests were performed on an Intel Core 2Duo 1.4 GHz CPU and 3GB RAM.

\section{Computational Results}

Instances for different scenarios were randomly generated to assess the behaviour of the model when the number of products increases (see Table1). Table 2 shows parameter values used to generate the testing problems.

Table 1. Analysed scenarios

\begin{tabular}{cccccc} 
Scenario & I & II & III & IV & V \\
\hline SKU [units] & 10 & 100 & 500 & 1000 & 5000 \\
\hline
\end{tabular}

Table 2. Parameter values for the numerical examples

\begin{tabular}{lc}
\hline Parameter & Value \\
\hline CostCar & 3 \\
CostShort & 50 \\
CostRecp & 5 \\
CostCapaS & 20 \\
CostCapaW & 3 \\
CostCapaFW & 10 \\
E $\left(U_{i}\right)$ & Uniform $[1,50]$ \\
$d_{i}^{L}$ & $N\left(\mu_{i}^{L}, \sigma_{i}^{L}\right)$ \\
\hline
\end{tabular}

The computational results for the different testing cases are shown in Table 3 . As it can be seen it was possible to analytically solve to optimality all the test scenarios in a very satisfactory computational time. In general the algorithm is very efficient and converge to the optimal solution in a very short time. Nevertheless, the computational time of LINGO rises as the problem size increases. 
Table 3. Model computational results

\begin{tabular}{cccccc} 
& \multicolumn{5}{c}{ Scenario } \\
\cline { 2 - 6 } & I & II & III & IV & V \\
\hline Total variables & 13 & 103 & 503 & 1003 & 5003 \\
Nonlinear variables $^{a}$ & 12 & 102 & 502 & 1002 & 5002 \\
Iterations & 203 & 647 & 1762 & 4020 & 16021 \\
CPU time $[m m: s s]$ & $00: 03$ & $00: 11$ & $00: 41$ & $01: 20$ & $14: 01$ \\
State & Global & Global & Global & Global & Global \\
& Opt. & Opt. & Opt. & Opt. & Opt. \\
\hline
\end{tabular}

${ }^{a}$ Variables involved in the nonlinear relationships of the model.

In order to point out some more features of the proposed model, more experiments were conducted for a warehouse problem with 500 SKUs (scenario III). Again parameter values from Table 2 were used and service levels between $95 \%$ and $99 \%$ were considered.

Table 4 shows that a private warehouse can be less expensive than renting all the storage capacity, even considering higher amounts of stock. When the privately operated warehouse has an upper storage limit, the total optimal storage capacity equals the optimal capacity for the strategy of using only a public warehouse. This happens whenever the storage upper limit of the private warehouse is less than the optimal storage solution of the public warehouse.

Table 4. Warehouse dimension and cost

\begin{tabular}{ccccc}
\hline$w$ & $M$ & CapaS & CapaW & Total Cost \\
\hline 0 & - & 694 & - & 51060 \\
& unlimited & 0 & 842 & 45724 \\
& 300 & 394 & 300 & 48960 \\
\multirow{2}{*}{1} & 500 & 194 & 500 & 47560 \\
& 600 & 94 & 600 & 46860 \\
& 700 & 0 & 700 & 46149 \\
& 900 & 0 & 842 & 45724 \\
\hline
\end{tabular}

Additional results also show that the inventory costs have a significant impact in warehouse management (see Table 5). As expected the integration of an appropriate inventory policy may result in a reduction of the total warehousing costs.

The gathered results also indicate that a mixed strategy (both owned and rented) would be better than renting all the storage space. Warehouse management should then achieve high levels of utilization for owned storage space and 
Table 5. Warehouse total costs

\begin{tabular}{ccccc}
\hline$w$ & $M$ & Inventory Costs & Warehouse Costs & Total Costs \\
\hline 0 & - & 23908 & 27152 & 51060 \\
& unlimited & 23878 & 21846 & 45724 \\
& 300 & 23908 & 25052 & 48960 \\
1 & 500 & 23908 & 23652 & 47560 \\
& 600 & 23908 & 22952 & 46860 \\
& 700 & 23903 & 22246 & 46149 \\
& 900 & 23878 & 21846 & 45724 \\
\hline
\end{tabular}

use additional rented space on a short-term basis to meet peak space requirements.

As it can be seen in Table 6, different cost elements have different impacts in warehouse total cost.

Table 6. Warehouse inventory and operational costs

\begin{tabular}{cccccc}
\hline$w$ & $M$ & CostCar & CostAqu & CostShort & CostRecp \\
\hline \multirow{2}{*}{0} & - & 1056 & 21436 & 1416 & 13272 \\
& unlimited & 1279 & 21436 & 1163 & 10900 \\
& 300 & 1056 & 21436 & 1416 & 13272 \\
\multirow{3}{*}{1} & 500 & 1056 & 21436 & 1416 & 13272 \\
& 600 & 1056 & 21436 & 1416 & 13272 \\
& 700 & 1065 & 21436 & 1402 & 13146 \\
& 900 & 1279 & 21436 & 1163 & 10900 \\
\hline
\end{tabular}

Since numerical tests were performed without having the real value of the different parameters involved in the model, and in order to highlight some relevant issues in the performance of the model, it is important to investigate how much influence some fluctuations in different cost elements may have in the optimal solution. For this purpose some of these costs will be selected and will vary $(-10 \%$ and $25 \%$ ) one at a time.

Denoting $x$ and $x^{*}$ as the initial parameter value and the new value, and $w(x)$ and $w\left(x^{*}\right)$ as the size of the storage capacity we will measure the impact on warehouse size of some variations using the following equation:

$$
\frac{w(x)-w\left(x^{*}\right)}{w\left(x^{*}\right)} \times 100 \% .
$$

Table 7 summarizes the impact of cost fluctuations in the warehouse optimal size. A detailed look at the different cost changes reveals that the reception cost 
(CostRecp) has a larger impact in warehouse size. As expected, an increase in this cost forces the model to increase the optimal order quantities and consequently the warehouse storage size increases. In a similar way decreasing the reception cost causes a decrease of the storage size area. On the other hand, the carrying cost (CostCar) and the shortage cost (CostShort) have lower impacts in the optimal solution with changes in warehouse size less than $1.2 \%$.

Table 7. Impact in warehouse size (\%)

\begin{tabular}{crrr} 
Parameter & Variation & CapaS & CapaW \\
\hline \multirow{2}{*}{ CostCar } & -10 & -0.71 & -0.59 \\
& 25 & 1.01 & 1.20 \\
\hline \multirow{2}{*}{ CostShort } & -10 & 0.58 & 0.48 \\
& 25 & -0.99 & -1.17 \\
\hline \multirow{2}{*}{ CostRecp } & -10 & 4.83 & 4.73 \\
& 25 & -9.51 & -9.55 \\
\hline \multirow{2}{*}{ CostCapaS } & -10 & -4.67 & - \\
& 25 & 10.86 & - \\
\hline \multirow{2}{*}{ CostCapaFW } & -10 & - & -3.55 \\
& 25 & - & 8.08 \\
\hline
\end{tabular}

The results also show that despite the inventory costs have a significant impact in the total warehouse costs, also variations in the warehouse costs mean significant impact in the storage size area. For example, the model is very sensitive to the external capacity cost (CostCapaS) where an increase of $25 \%$ causes a reduction of $10.86 \%$ of the rented area. In a similar way a $25 \%$ increase in the cost of the owned warehouse (CostCapaFW) causes an reduction of $8.08 \%$ of the size storage area.

The fluctuations that were carried out in some of the parameters of the model revealed that the size of the warehouse is more sensitive to changes of some parameters than to others. Nonetheless, the warehouse size solution is quite robust once the proposed model integrates the different costs in a way that balances the trade-offs among them, minimizing the effect of the change.

Finally it should be noted that in our numerical results the parameter variations were done one at a time. Future research might also be performed to investigate joint effects of different parameters on the quality of the solution of the model.

\section{Conclusions and Future Work}

Most of the times, inventory decisions and warehouse design decisions are independently considered. In fact, a single decision model that integrates several 
decisions concerning warehouse design and planning is very complex due to the tremendous amount of information to be processed, to the large number of existing alternatives, to the existence of various and often conflicting objectives and to the uncertainty inherent in the material flow into, through and out of the warehouse.

Throughout this work our aim was to show the value of integrating inventory decisions and warehouse design decisions, in particular the strategic decision that concerns the size of a warehouse. For that purpose a mathematical programming model was proposed and discussed.

The proposed mathematical programming model jointly integrates: (i) the size of a private storage warehouse; (ii) the external additional storage capacity, if needed; and (iii) the replenishment quantities and reorder points of the products.

Although the model is a mixed-integer nonlinear programming model with a large number of variables, usually difficult to solve by general optimization packages, it was possible to solve to optimality some test scenarios in a very satisfactory computational time. Nevertheless for large instances the computational time increases considerably.

Computational results were obtained for five different scenarios randomly generated. The gathered results also suggest that, for this particular situation, a mixed strategy, both owned and rented storage capacity, would have lower total costs than renting all the storage space. High levels of utilization should be achieved for owned storage space and additional rented space should be used to meet peak space requirements. It has also been shown that inventory costs have a significant impact in warehouse system costs.

A sensitivity analysis was performed to observe the impact of cost fluctuations in the warehouse optimal size. For this purpose some costs were selected and varied one at a time. The results show that the size of the warehouse is more sensitive to changes of some parameters than to others.

Even though the presented model integrates two important decisions concerning the design of a warehouse, many other decisions were not included. For example the size of the functional areas inside the warehouse; the assignment and allocation problem of the products; the picking and routing strategies, etc.

In summary, despite some advances in integrated approaches, further research focusing integrated models where different processes in the warehouse are jointly considered (and its corresponding dynamic nature), is still required. Given the prevalence of warehouses in the supply chain networks we believe that such research achievements can have a significant impact in the supply chain performance.

\section{References}

1. Bartholdi, J., Hackman, S.: Warehouse \& Distribution Science (2006). Release 0.76, http://www. warehouse-science.com

2. Geraldes, C., Carvalho, S., Pereira, G.: A warehouse design decision model. In: Proceedings of the International Engineering Management Conference 2008, IEMCEurope 2008, Estoril, Portugal (2008) ISBN: 978-1-4244-2289-0, IEEE Catalog Number: CFP08EMS 
3. Ghiani, G., Laporte, G., Musmanno, R.: Introduction to Logistics Systems Planning and Control. John Wiley \& Sons Ltd, England (2004)

4. Gu, J., Goetschalckx, M., McGinnis, L.F.: Research on warehouse operation: A comprehensive review. European Journal of Operational Research 177, 1-21 (2007)

5. Gray, A., Karmarkar, U., Seidman, A.: Design and operation of an orderconsolidation warehouse: models and application. European Journal of Operational Research 58, 14-36 (1992)

6. Hadley, G., Whitin, T.: Analysis of Inventory Systems. Prentice-Hall, Englewood Cliffs (1963)

7. Hassan, M.: A framework for the design of warehouse layout. Facilities 20(13/14), 432-440 (2002)

8. Heragu, S., Du, L., Mantel, R.J., Schuur, P.C.: Mathematical model for warehouse design and product allocation. International Journal of Production Research 43(2), 432-440 (2005)

9. Nahmias, S.: Production and Operation Analysis, 3rd edn. McGraw-Hill International Editions (1997)

10. Rouwenhorst, B., Reuter, B., Stokrahm, V., van Houtum, G., Mantel, R., Zijm, W.: Warehouse design and control: Framework and literature review. European Journal of Operational Research 122, 515-533 (2000)

11. Strack, G., Pochet, Y.: An integrated model for warehouse design and planning. European Journal of Operational Research 204, 35-50 (2010)

12. Tompkins, J.A., White, J.A., Bozer, Y.A., Tanchoco, J.M.A.: Facilities Planning. John Wiley \& Sons, New York (2003)

13. Van den Berg, J., Sharp, G., Gademann, A., Pochet, Y.: Forward-reserve allocation in a warehouse with unit-load replenishments. European Journal of Operational Research 111, 98-113 (1998)

14. Van den Berg, J., Zjim, W.: Models for warehouse management: Classification and examples. International Journal of Production Economics 59, 519-528 (1999)

15. Van den Berg, J.: A literature survey on planning and control of warehousing systems. IEEE Transactions 31, 751-762 (1999) 